\title{
New factorization relations for nonlinear sigma model amplitudes
}

\author{
N. E. J. Bjerrum-Bohr, ${ }^{1}$ Humberto Gomez, ${ }^{1,2}$ and Andreas Helset ${ }^{1}$ \\ ${ }^{1}$ Niels Bohr International Academy and Discovery Center Niels Bohr Insitute, \\ University of Copenhagen Blegdamsvej 17, DK-2100 Copenhagen Ø, Denmark \\ ${ }^{2}$ Facultad de Ciencias, Basicas Universidad Santiago de Cali, \\ Calle $5 N^{\circ}$ 62-00 Barrio Pampalinda Cali, Valle, Colombia
}

(Received 3 December 2018; published 19 February 2019)

\begin{abstract}
We obtain novel factorization identities for nonlinear sigma model amplitudes using a new integrand in the Cachazo-He-Yuan double-cover prescription. We find that it is possible to write very compact relations using only longitudinal degrees of freedom. We discuss implications for on shell recursion.
\end{abstract}

DOI: 10.1103/PhysRevD.99.045009

\section{INTRODUCTION}

Cachazo, He and Yuan (CHY) invented in Ref. [1] a new method for calculating S-matrix elements. This formalism has numerous applications and many interesting connections, see for instance Refs. [2-4]. The CHY construction was formally proven by Dolan and Goddard in Ref. [5].

The main ingredients are the $n$-point scattering equations

$0=S_{a} \equiv \sum_{b=1, b \neq a}^{n} \frac{s_{a b}}{z_{a b}}, \quad z_{a b} \equiv z_{a}-z_{b}, \quad s_{a b} \equiv 2 k_{a} \cdot k_{b}$,

where $z_{a}$ are auxiliary variables on the Riemann sphere and $k_{a}$ are momenta. In the CHY formalism one has to integrate over a contour containing the $(n-3)$ ! independent solutions of the scattering equations.

As computations in the $\mathrm{CHY}$ formalism grow factorially in complexity with $n$, integration rules have been developed at tree [6-9] and loop level [10], so that analytical results for amplitudes can be derived without solving the scattering equations explicitly.

Recently, the CHY formalism was reformulated by one of us in the context of a double cover [11] (called the " $\Lambda$-formalism" in Refs. [11,12]). Here, the basic variables are elements of $\mathbb{C P}^{2}$, and not $\mathbb{C P}^{1}$ as in the original $\mathrm{CHY}$ formalism. One advantage of the extra machinery is that amplitudes in the double-cover formulation naturally factorize into smaller $\mathbb{C P}^{1}$ pieces, and this is a useful laboratory for deriving new amplitude identities.

We will start by reviewing the CHY formalism for the nonlinear sigma model (NLSM) and provide an alternative

Published by the American Physical Society under the terms of the Creative Commons Attribution 4.0 International license. Further distribution of this work must maintain attribution to the author(s) and the published article's title, journal citation, and DOI. Funded by SCOAP. formulation that employs a new integrand. Next, we will show how the double-cover formalism naturally factorizes this new CHY formulation in a surprising way.

\section{A NEW CHY INTEGRAND}

As explained in Ref. [13], the flavor-ordered partial $U(N)$ nonlinear sigma model amplitude in the scattering equation framework is given by the contour integral

$$
\begin{aligned}
A_{n}(\alpha) & =\int d \mu_{n} H_{n}(\alpha), \\
d \mu_{n} & \equiv\left(z_{i j} z_{j k} z_{k i}\right)^{2} \prod_{\substack{a=1 \\
a \neq\{i, j, k\}}}^{n} \frac{d z_{a}}{S_{a}},
\end{aligned}
$$

where $(\alpha)=(\alpha(1), \ldots, \alpha(n))$ denotes a partial ordering. The integrand is given by

$$
\begin{aligned}
H_{n}(\alpha) & =\operatorname{PT}(\alpha) \times\left(\mathrm{Pf}^{\prime} \mathrm{A}\right)^{2} \\
\operatorname{PT}(\alpha) & \equiv \frac{1}{z_{\alpha(1) \alpha(2)} z_{\alpha(2) \alpha(3)} \cdots z_{\alpha(n) \alpha(1)}}, \\
\operatorname{Pf}^{\prime} \mathrm{A} & \equiv \frac{(-1)^{i+j}}{z_{i j}} \operatorname{Pf}\left[(\mathbf{A})_{i j}^{i j}\right] .
\end{aligned}
$$

Here $\operatorname{PT}(\alpha)$ and $\mathrm{Pf}^{\prime} \mathrm{A}$ are the Parke-Taylor (PT) factor and the reduced Pfaffian of matrix A, respectively. The $n \times n$ antisymmetric matrix, $A$, is defined as,

$\mathrm{A}_{a b} \equiv \frac{s_{a b}}{z_{a b}} \quad$ for $a \neq b, \quad$ and $\quad \mathrm{A}_{a b} \equiv 0 \quad$ for $a=b$.

In general, $(\mathbf{A})_{j_{1} \cdots j_{p}}^{i_{1} \cdots i_{p}}$ denotes the reduced matrix obtained by removing the rows, $\left\{i_{1}, \ldots, i_{p}\right\}$, and columns, $\left\{j_{1}, \ldots, j_{p}\right\}$, 
from $A$. Note that when the number of external particles $n$ is odd, $\mathrm{Pf}^{\prime} \mathrm{A}=0$, and $A_{n}(\alpha)$ vanishes.

Using Eq. (5), we have

$$
\left(\operatorname{Pf}^{\prime} \mathbf{A}\right)^{2}=\frac{(-1)^{i+j+m+p}}{z_{i j} z_{m p}} \operatorname{Pf}\left[(\mathbf{A})_{i j}^{i j}\right] \times \operatorname{Pf}\left[(\mathbf{A})_{m p}^{m p}\right] .
$$

With the choice $\{i, j\}=\{m, p\}$, this product of Pfaffians becomes a determinant,

$$
\left(\mathrm{Pf}^{\prime} \mathrm{A}\right)^{2}=-\mathrm{PT}(m, p) \operatorname{det}\left[(\mathrm{A})_{m p}^{m p}\right] .
$$

We will now discuss the following new matrix identities. On the support of the scattering equations and the massless condition, $\left\{S_{a}=0, k_{a}^{2}=0\right\}$, we find when $m \neq p \neq q$

$$
\begin{aligned}
& \operatorname{Pf}\left[(\mathbf{A})_{m p}^{m p}\right] \times \operatorname{Pf}\left[(\mathbf{A})_{p q}^{p q}\right]=\operatorname{det}\left[(\mathbf{A})_{p q}^{m p}\right], \\
& \operatorname{det}\left[(\mathbf{A})_{p q}^{m p}\right]=0 \quad \text { if } n \text { odd } .
\end{aligned}
$$

A proof of these identities will be provided in Ref. [14]. Using the non-anti-symmetric matrix, $(\mathrm{A})_{j k}^{i j}$, we define the objects (with $i<j<k$ )

$$
\begin{aligned}
A_{n}^{\prime}(\alpha) & =\int d \mu_{n} \mathrm{PT}(\alpha) \frac{(-1)^{i+k}}{z_{i j} z_{j k}} \operatorname{det}\left[(\mathrm{A})_{j k}^{i j}\right], \\
A_{n}^{(i j)}(\alpha) & =\int d \mu_{n} \mathrm{PT}(\alpha) \frac{(-1)^{i+j}}{z_{i j}} \operatorname{det}\left[(\mathrm{A})_{j}^{i}\right] .
\end{aligned}
$$

Note that in Eqs. (11) and (12) we have reduced the A matrix with the indices $\{i, j, k\}$ associated with the Faddeev-Popov determinant. This gauge choice will be convenient later. We now have the following equality,

$$
A_{n}^{\prime}(\alpha)=A_{n}(\alpha)
$$

when all particles are on shell. When there are off shell particles, the identity is true only if the number of particles is even. When the number of particles is odd and there are off shell particles, one has $A_{n}(\alpha)=0$ while $A_{n}^{\prime}(\alpha) \neq 0$. Since the A matrix has the corank 2 on the support of the scattering equations and the massless condition, $\left\{S_{a}=0, k_{a}^{2}=0\right\}, A_{n}^{(i j)}(\alpha)$ vanishes trivially. However, when there are off shell particles the amplitude $A_{n}^{(i j)}(\alpha)$ is no longer zero.

These observations will be crucial in obtaining the new factorization relations.

\section{THE DOUBLE-COVER REPRESENTATION}

In the double-cover version of the CHY construction, the $n$-point amplitude is given as a contour integral on the double-covered Riemann sphere with $n$ punctures.
The pairs $\left(\sigma_{1}, y_{1}\right),\left(\sigma_{2}, y_{2}\right), \ldots,\left(\sigma_{n}, y_{n}\right)$ provide the new set of doubled variables restricted to the curves

$$
0=C_{a} \equiv y_{a}^{2}-\sigma_{a}^{2}+\Lambda^{2} \quad \text { for } a=1, \ldots, n .
$$

A translation table has been worked out in detail in Ref. [11]. The double-cover formulation of the NLSM is given by the integral

$$
\begin{aligned}
A_{n}(\alpha) & =\int_{\Gamma} d \mu_{n}^{\Lambda} \frac{(-1) \Delta(i j k) \Delta(i j k \mid r)}{S_{r}^{\tau}} \times \mathcal{I}_{n}(\alpha), \\
d \mu_{n}^{\Lambda} & \equiv \frac{1}{2^{2}} \frac{d \Lambda}{\Lambda} \prod_{a=1}^{n} \frac{y_{a} d y_{a}}{C_{a}} \times \prod_{\substack{d=1 \\
d \neq i, j, k}}^{n} \frac{d \sigma_{d}}{S_{d}^{\tau}}, \\
\tau(a, b) & \equiv \frac{1}{2 \sigma_{a b}}\left(\frac{y_{a}+y_{b}+\sigma_{a b}}{y_{a}}\right), \quad S_{a}^{\tau} \equiv \sum_{\substack{b=1 \\
b \neq a}}^{n} s_{a b} \tau(a, b), \\
\Delta(i j k) & \equiv(\tau(i, j) \tau(j, k) \tau(k, i))^{-1}, \\
\Delta(i j k \mid r) & \equiv \sigma_{i} \Delta(j k r)-\sigma_{r} \Delta(i j k)+\sigma_{k} \Delta(r i j)-\sigma_{j} \Delta(k r i) .
\end{aligned}
$$

The $\Gamma$ contour is defined by the $2 n-3$ equations

$$
\Lambda=0, \quad S_{d}^{\tau}(\sigma, y)=0, \quad C_{a}=0,
$$

for $d \neq\{i, j, k, r\}$ and $a=1, \ldots, n$.

The integrand is given by

$\mathcal{I}_{n}(\alpha)=-\mathrm{PT}^{\tau}(\alpha) \prod_{a=1}^{n} \frac{(y \sigma)_{a}}{y_{a}} \mathrm{PT}^{T}(m, p) \operatorname{det}\left[\left(\mathrm{A}^{\Lambda}\right)_{m p}^{m p}\right]$,

where $(y \sigma)_{a} \equiv y_{a}+\sigma_{a}$. To obtain the kinematic matrix and the Parke-Taylor factors we need to do the following replacements

$$
\begin{aligned}
\mathrm{A} \rightarrow \mathrm{A}^{\Lambda}, & \text { and } \quad \mathrm{PT} \rightarrow \mathrm{PT}^{T} \quad \text { for } z_{a b} \rightarrow T_{a b}^{-1}, \\
\mathrm{PT} & \rightarrow \mathrm{PT}^{\tau} \quad \text { for } z_{a b} \rightarrow \tau(a, b)^{-1},
\end{aligned}
$$

with $T_{a b} \equiv \frac{1}{(y \sigma)_{a}-(y \sigma)_{b}}$. Analogous to Eq. (11), we can now write down a new form for the integrand

$\mathcal{I}_{n}^{\prime}(\alpha)=\mathrm{PT}^{\tau}(\alpha) \prod_{a=1}^{n} \frac{(y \sigma)_{a}}{y_{a}}(-1)^{i+k} T_{i j} T_{j k} \operatorname{det}\left[\left(\mathrm{A}^{\Lambda}\right)_{j k}^{i j}\right]$,

where $\{i, j, k\}$ are the same labels as in $\Delta(i j k) \Delta(i j k \mid r)$. For more details on the double-cover prescription, see Refs. [11,14,15]. 


\section{FACTORIZATION}

Let us start by considering the four-point amplitude, $A_{4}^{\prime}(1,2,3,4)$, with the gauge fixing $(i j k \mid r)=(123 \mid 4)$. We will denote sums of cyclically-consecutive external momenta (modulo the total number of external momenta) by $P_{i: j} \equiv k_{i}+k_{i+1}+\cdots+k_{j-1}+k_{j}$,. For expressions involving only two (not necessarily consecutive) momenta, we are using the shorthand notation $P_{i j} \equiv k_{i}+k_{j}$. We focus on the configuration where the sets of punctures $\left(\sigma_{1}, \sigma_{2}\right)$ and $\left(\sigma_{3}, \sigma_{4}\right)$ are respectably on the upper and the lower sheet of the curves

$$
\begin{array}{ll}
\left(y_{1}=+\sqrt{\sigma_{1}^{2}-\Lambda^{2}}, \sigma_{1}\right), & \left(y_{2}=+\sqrt{\sigma_{2}^{2}-\Lambda^{2}}, \sigma_{2}\right), \\
\left(y_{3}=-\sqrt{\sigma_{3}^{2}-\Lambda^{2}}, \sigma_{3}\right), & \left(y_{4}=-\sqrt{\sigma_{4}^{2}-\Lambda^{2}}, \sigma_{4}\right) .
\end{array}
$$

Expanding all elements in $A_{4}^{\prime}(1,2,3,4)$ around $\Lambda=0$, we obtain (to leading order)

$$
\begin{aligned}
&\left.\mathrm{PT}^{\tau}(1,2,3,4)\right|_{3,4} ^{1,2}= \frac{\Lambda^{2}}{2^{2}} \frac{1}{\left(\sigma_{12} \sigma_{2 P_{34}} \sigma_{P_{34}}\right)} \frac{1}{\left(\sigma_{P_{12} 3} \sigma_{34} \sigma_{4 P_{12}}\right)}, \\
&\left.\frac{\Delta(123) \Delta(123 \mid 4)}{S_{4}^{\tau}}\right|_{3,4} ^{1,2}= \frac{2^{5}}{\Lambda^{4}}\left(\sigma_{12} \sigma_{2 P_{34}} \sigma_{P_{34}}\right)^{2}\left(\frac{1}{s_{34}}\right) \\
& \times\left(\sigma_{P_{12} 3} \sigma_{34} \sigma_{4 P_{12}}\right)^{2}, \\
&\left.\prod_{a=1}^{4} \frac{(y \sigma)_{a}}{y_{a}} T_{12} T_{23} \operatorname{det}\left[\left(\mathrm{A}^{\Lambda}\right)_{23}^{12}\right]\right|_{3,4} ^{1,2} \\
&=\frac{\Lambda^{2}}{2^{2}} \frac{1}{\sigma_{12} \sigma_{2 P_{34}}} \frac{s_{14}}{\sigma_{P_{34}}} \frac{1}{\sigma_{P_{12} 3}} \frac{(-1) s_{34}}{\sigma_{34} \sigma_{4 P_{12}}},
\end{aligned}
$$

where we have introduced the new fixed punctures $\sigma_{P_{34}}=\sigma_{P_{12}}=0$. Since we want to arrive at factorization identity for nonlinear sigma model amplitudes (inspired by previous work for Yang-Mills theories, see Ref. [15]) we are now going to introduce polarizations associated with the punctures, $\sigma_{P_{34}}=\sigma_{P_{12}}=0$, i.e., $\epsilon_{34}^{M}$ and $\epsilon_{12}^{M}$. Thus,

$$
\begin{aligned}
s_{14} & =2\left(k_{1} \cdot k_{4}\right)=2\left(k_{1 \mu} \times \eta^{\mu \nu} \times k_{4 \nu}\right) \\
& =\sum_{M}\left(\sqrt{2} k_{1} \cdot \epsilon_{34}^{M}\right) \times\left(\sqrt{2} k_{4} \cdot \epsilon_{12}^{M}\right),
\end{aligned}
$$

employing,

$$
\sum_{M} \epsilon_{i}^{M \mu} \epsilon_{j}^{M \nu}=\eta^{\mu \nu}
$$

After separating the labels $\{1,2\}$ and $\{3,4\}$, it is simple to rearrange Eq. (24) as a product of two reduced determinants,

$$
\begin{aligned}
& \frac{1}{\sigma_{12} \sigma_{2 P_{34}}} \frac{\left(\sqrt{2} k_{1} \cdot \epsilon_{34}^{M}\right)}{\sigma_{P_{34}}}=\frac{1}{\sigma_{12} \sigma_{2 P_{34}}} \operatorname{det}\left[\frac{\sqrt{2} k_{1} \cdot \epsilon_{34}^{M}}{\sigma_{P_{34}}}\right], \\
& \frac{(-1)}{\sigma_{P_{12} 3}} \frac{s_{34}\left(\sqrt{2} k_{4} \cdot \epsilon_{12}^{M}\right)}{\sigma_{34} \sigma_{4 P_{12}}}=\frac{(-1)}{\sigma_{P_{12} 3}} \operatorname{det}\left[\begin{array}{cc}
\frac{\sqrt{2} k_{3} \cdot \epsilon_{12}^{M}}{\sigma_{3 P_{12}}} & \frac{s_{34}}{\sigma_{34}} \\
\frac{\sqrt{2} k_{4} \cdot \epsilon_{12}^{M}}{\sigma_{4 P_{12}}} & 0
\end{array}\right],
\end{aligned}
$$

therefore

$$
\begin{aligned}
\prod_{a=1}^{4} & \left.\frac{(y \sigma)_{a}}{y_{a}} T_{12} T_{23} \operatorname{det}\left[\left(\mathrm{A}^{\Lambda}\right)_{23}^{12}\right]\right]_{3,4}^{1,2} \\
= & -\frac{\Lambda^{2}}{2^{2}} \times \sum_{M} \frac{1}{\sigma_{12} \sigma_{2 P_{34}}} \times \operatorname{det}\left[\frac{\sqrt{2} k_{1} \cdot \epsilon_{34}^{M}}{\sigma_{P_{34} 1}}\right] \\
& \times \frac{(-1)}{\sigma_{P_{12} 3}} \operatorname{det}\left[\begin{array}{cc}
\frac{\sqrt{2} k_{3} \cdot \epsilon_{12}^{M}}{\sigma_{3 P_{12}}} & \frac{s_{34}}{\sigma_{34}} \\
\frac{\sqrt{2} k_{4} \cdot \epsilon_{12}^{M}}{\sigma_{4 P_{12}}} & 0
\end{array}\right] .
\end{aligned}
$$

The new matrices in Eq. (27) can be obtained from the A matrix by replacing the off shell momenta, $P_{34}$ and $P_{12}$, by their corresponding off shell polarization vectors,

$$
\begin{aligned}
& \operatorname{det}\left[(\mathrm{A})_{2 P_{34}^{12}}^{12}\right] \rightarrow \operatorname{det}\left[\frac{\sqrt{2} k_{1} \cdot \epsilon_{34}^{M}}{\sigma_{P_{34}}}\right] \quad \text { for } P_{34} \rightarrow \frac{1}{\sqrt{2}} \epsilon_{34}^{M}, \\
& \operatorname{det}\left[(\mathrm{A})_{3}^{P_{12}}\right] \rightarrow \operatorname{det}\left[\begin{array}{cc}
\frac{\sqrt{2} k_{3} \cdot \epsilon_{12}^{M}}{\sigma_{3 P_{12}}} & \frac{s_{34}}{\sigma_{34}} \\
\frac{\sqrt{2} k_{4} \cdot \epsilon_{12}^{M}}{\sigma_{4 P_{12}}} & 0
\end{array}\right] \text { for } P_{12} \rightarrow \frac{1}{\sqrt{2}} \epsilon_{12}^{M},
\end{aligned}
$$

where the A matrix in Eq. (28) is the $3 \times 3$ matrix related with the punctures $\left(\sigma_{1}, \sigma_{2}, \sigma_{P_{34}}\right)$, while the matrix in Eq. (29) corresponds to the punctures $\left(\sigma_{P_{12}}, \sigma_{3}, \sigma_{4}\right)$.

Using the measure, $d \mu_{4}^{\Lambda}=\frac{1}{2^{2}} \frac{d \Lambda}{\Lambda}$, we now perform the $\Lambda$ integral and the amplitude becomes

$$
\begin{aligned}
& \left.A_{4}^{\prime}(1,2,3,4)\right|_{3,4} ^{1,2} \\
& \quad=\frac{1}{2} \sum_{M} \frac{A_{3}^{\prime}\left(1,2, P_{34}^{\epsilon^{M}}\right) \times A_{3}^{\left(P_{12} 3\right)}\left(P_{12}^{\epsilon^{M}}, 3,4\right)}{s_{12}}=\frac{s_{14}}{2},
\end{aligned}
$$

where the notation, $P_{i}^{\epsilon^{M}}$, means one must make the replacement, $P_{i} \rightarrow \frac{1}{\sqrt{2}} \epsilon_{i}^{M}$, and use Eq. (26). The overall factor $1 / 2$ cancels out after summing over mirrored configurations, i.e., $\left.A_{4}^{\prime}(1,2,3,4)\right|_{3,4} ^{1,2}+\left.A_{4}^{\prime}(1,2,3,4)\right|_{1,2} ^{3,4}=s_{14}$. Following the integration rules in Ref. [15], we also have the contribution (up to summing over mirrored configurations)

$$
\begin{aligned}
& \left.A_{4}^{\prime}(1,2,3,4)\right|_{2,3} ^{4,1} \\
& \quad=\frac{1}{2} \sum_{M} \frac{A_{3}^{\left(1 P_{23}\right)}\left(1, P_{23}^{\epsilon^{M}}, 4\right) \times A_{3}^{\prime}\left(P_{41}^{\epsilon^{M}}, 2,3\right)}{s_{14}}=\frac{s_{12}}{2} .
\end{aligned}
$$


Thus, the final result is

$$
\begin{aligned}
A_{4}^{\prime}(1,2,3,4)= & \sum_{M}\left[\frac{A_{3}^{\prime}\left(1,2, P_{34}^{\epsilon^{M}}\right) \times A_{3}^{\left(P_{12} 3\right)}\left(P_{12}^{\epsilon^{M}}, 3,4\right)}{s_{12}}\right. \\
& \left.+\frac{A_{3}^{\left(1 P_{23}\right)}\left(1, P_{23}^{\epsilon^{M}}, 4\right) \times A_{3}^{\prime}\left(P_{41}^{\epsilon^{M}}, 2,3\right)}{s_{14}}\right]=-s_{13} .
\end{aligned}
$$

The four-point amplitude is factorized in terms of threepoint functions. The general three-point functions where some or all particles can be off shell, are

$$
\begin{aligned}
A_{3}^{\prime}\left(P_{a}, P_{b}, P_{c}\right) & =s_{P_{c} P_{a}}=-\left(P_{a}^{2}-P_{b}^{2}+P_{c}^{2}\right), \\
A_{3}^{\left(P_{a} P_{b}\right)}\left(P_{a}, P_{b}, P_{c}\right) & =s_{P_{b} P_{c}} s_{P_{c} P_{a}} \\
& =\left(P_{c}^{2}-P_{a}^{2}+P_{b}^{2}\right)\left(P_{a}^{2}-P_{b}^{2}+P_{c}^{2}\right) .
\end{aligned}
$$

Since the nonlinear sigma model is a scalar theory it is an interesting proposition to consider longitudinal degrees of freedom only

$$
\sum_{L} \epsilon_{i}^{L \mu} \epsilon_{j}^{L \nu}=\frac{k_{i}^{\mu} k_{j}^{\nu}}{k_{i} \cdot k_{j}}
$$

Doing so we arrive at the equation

$$
\begin{aligned}
A_{4}^{\prime}(1,2,3,4)= & 2 \sum_{L}\left[(-1)^{3} \frac{A_{3}^{\prime}\left(1,2, P_{34}^{\epsilon^{L}}\right) \times A_{3}^{\left(P_{12} 3\right)}\left(P_{12}^{\epsilon^{L}}, 3,4\right)}{s_{12}}\right. \\
& \left.+(-1)^{3} \frac{A_{3}^{\prime}\left(P_{41}^{\epsilon_{1}^{L}}, 2,3\right) \times A_{3}^{\left(1 P_{23}\right)}\left(1, P_{23}^{\epsilon^{L}}, 4\right)}{s_{14}}\right] \\
& =-s_{13} .
\end{aligned}
$$

Surprisingly, it is possible to generalize this equation to higher point amplitudes. Here the overall sign of each contribution depends of the number of points of the subamplitudes. In Ref. [14], we will give more details on this phenomenon.

\section{NEW RELATIONS}

As will be shown in great detail elsewhere [14], using the double-cover prescription for a partial nonlinear sigma model amplitude one is led to the following general formula where an $n$-point amplitude is factorized into a product of two (single-cover) lower-point amplitudes:

$$
\begin{aligned}
& A_{n}^{\prime}(1,2,3,4, \ldots, n) \\
& =\sum_{i=4, M}^{n} \frac{A_{n-i+3}^{\prime}\left(1,2, P_{3: i}^{\epsilon^{M}}, i+1, \ldots n\right) A_{i-1}^{\left(P_{i+1: 2} 3\right)}\left(P_{i+1: 2}^{\epsilon^{M}}, 3, \ldots i\right)}{P_{i+1: 2}^{2}} \\
& +\sum_{M} \frac{A_{3}^{\prime}\left(P_{4: 1}^{\epsilon^{M}}, 2,3\right) \times A_{n-1}^{\left(1 P_{23}\right)}\left(1, P_{23}^{\epsilon^{M}}, 4, \ldots, n\right)}{P_{23}^{2}}
\end{aligned}
$$

Here $n$ is an even integer and we have used Eq. (26). The above expression is valid using the Möbius and scaleinvariance gauge choice $(i j k \mid r)=(123 \mid 4)$.

From the decomposition obtained by the double-cover method in Eq. (37), we are able to write down a new factorization relation, where only longitudinal degrees of freedom contribute,

$$
\begin{aligned}
& A_{n}^{\prime}(1,2,3,4, \ldots, n) \\
& =2\left[\sum_{i=4, L}^{n}(-1)^{i-1}\right. \\
& \quad \times \frac{A_{n-i+3}^{\prime}\left(1,2, P_{3: i}^{\epsilon^{L}}, i+1, \ldots n\right) \times A_{i-1}^{\left(P_{i+1: 2} 3\right)}\left(P_{i+1: 2}^{\epsilon^{L}}, 3, \ldots i\right)}{P_{i+1: 2}^{2}} \\
& \left.\quad+\sum_{L}(-1)^{3} \frac{A_{3}^{\prime}\left(P_{4: 1}^{\epsilon^{L}}, 2,3\right) \times A_{n-1}^{\left(1 P_{23}\right)}\left(1, P_{23}^{\epsilon^{L}}, 4, \ldots, n\right)}{P_{23}^{2}}\right],
\end{aligned}
$$

where Eq. (35) was used. We checked this formula up to ten points.

Since the above factorization relation includes only longitudinal contributions, we can rewrite it in a more elegant form, involving only the $A_{q}^{\prime}$ amplitudes. Using the definitions given in Eqs. (11)-(12) and under the gauge fixing $(i j k)$, with $i<j<k$, we have the following two identities [14]

$A_{q}^{(i j)}\left(\ldots, P_{i}, \ldots\right)=P_{i}^{2} A_{q}^{\prime}\left(\ldots, P_{i}, \ldots\right), \quad q=2 m+1$

$A_{q}^{(i j)}\left(\ldots, P_{i}, \ldots\right)=-P_{i}^{2} A_{q}^{\prime}\left(\ldots, P_{i}, \ldots\right), \quad q=2 m$,

where $P_{i}^{2} \neq 0$. In addition, $A_{q}^{(i j)}$ satisfies the useful identities

$$
\begin{aligned}
A_{q}^{(i j)}\left(1, \ldots, i, \ldots P_{j}, \ldots k \ldots q\right) & =A_{q}^{(j k)}\left(1, \ldots, i, \ldots P_{j}, \ldots k \ldots q\right) \\
A_{q}^{(i j)}\left(1 \ldots, \ldots, \ldots P_{j}, \ldots k \ldots q\right) & =A_{q}^{(i j)}\left(2 \ldots i \ldots P_{j} \ldots k \ldots q, 1\right) \\
& =\ldots=A_{q}^{(j k)}\left(\ldots P_{j} \ldots k \ldots q \ldots i\right) \\
& =A_{q}^{(i j)}\left(\ldots k \ldots q, 1, \ldots i \ldots P_{j} \ldots\right) .
\end{aligned}
$$

Applying the identities Eqs. (39)-(40), it is straightforward to obtain

$$
\begin{aligned}
& A_{n}^{\prime}(1,2,3,4, \ldots, n) \\
& =\sum_{i=4}^{n} \frac{A_{n-i+3}^{\prime}\left(1,2, P_{3: i}, i+1, \ldots n\right) \times A_{i-1}^{\prime}\left(P_{i+1: 2}, 3, \ldots i\right)}{P_{i+1: 2}^{2}} \\
& \quad+\frac{A_{3}^{\prime}\left(P_{4: 1}, 2,3\right) \times A_{n-1}^{\prime}\left(P_{23}, 4, \ldots, n, 1\right)}{P_{23}^{2}}
\end{aligned}
$$


where the factorization formula has been written in terms of the generalized amplitude $A_{q}^{\prime}$. Other gauge choices will naturally with lead to alternative factorization formulas.

\section{A. BCFW recursion}

It is interesting to analyze the new factorization identities in comparison with expressions originating from the BrittoCachazo-Feng-Witten (BCFW) recursion [16]. We introduce the momentum deformation

$$
k_{2}^{\mu}(z)=k_{2}^{\mu}+z q^{\mu}, k_{3}^{\mu}(z)=k_{3}^{\mu}-z q^{\mu}, z \in \mathbb{C},
$$

where $q^{\mu}$ satisfies $k_{2} \cdot q=k_{3} \cdot q=q \cdot q=0$. Deformed momenta are conserved and on shell: $k_{1}+k_{2}(z)+k_{3}(z)+$ $k_{4}+\cdots+k_{n}=0$ and $k_{2}^{2}(z)=k_{3}^{2}(z)=0$. We consider the general amplitude, $A_{n}(1, \ldots, n)$, where $n$ is an even integer. From Eq. (41) using Cauchy's theorem we have

$$
\begin{aligned}
A_{n}(1,2, \ldots, n) \\
=-\sum_{i=3}^{n / 2} \operatorname{Res}_{P_{2 i: 2}^{2}(z)=0}\left[A_{n-2 i+4}^{\prime}\left(1,2, P_{3: 2 i-1}, 2 i, \ldots, n\right)\right. \\
\left.\quad \times \frac{A_{2 i-2}^{\prime}\left(P_{2 i: 2}, 3, \ldots, 2 i-1\right)}{z P_{2 i: 2}^{2}(z)}\right] \\
\quad-\operatorname{Res}_{z=\infty}\left[\frac{A_{n}^{\prime}(1,2, \ldots, n)(z)}{z}\right]
\end{aligned}
$$

Only the even amplitudes, namely $A_{2 q}^{\prime}$, contribute to the physical residues. This is simple to understand as we have the identity, $A_{2 q}(1, \ldots, 2 q)=A_{2 q}^{\prime}(1, \ldots, 2 q)$, so only subamplitudes with an even number of particles produce physical factorization channels. On the other hand, when the number of particles is odd, the off shell $\left(P_{i}^{2} \neq 0\right)$ amplitude, $A_{2 q+1}^{\prime}\left(\ldots, P_{i}, \ldots\right)$, is proportional to $P_{i}^{2}$, since it must vanish when all particles are on shell. So, the poles, $P_{2 i-1: 2}^{2}, i=3, \ldots, \frac{n}{2}+1$ and $P_{23}$, are all spurious and the subamplitudes with an odd number of particles only contribute the boundary term at $z=\infty$.

Finally, it is important to remark that after evaluating the residues, $P_{2 i: 2}^{2}(z)=0$, in Eq. (43), one obtains extra nonphysical contributions, which cancel out combining with terms associated with the residue at $z=\infty$. Therefore, the effective boundary contribution is just given by the subamplitudes with an odd number of particles

$$
\begin{aligned}
\operatorname{Res}_{z=\infty} & {\left[\frac{A_{n}^{\prime}(1,2, \ldots, n)(z)}{z}\right]^{\text {Effective }} } \\
= & \partial_{\frac{1}{z}}\left[\sum_{i=3}^{n / 2+1} A_{n-2 i+5}^{\prime}\left(1,2, P_{3: 2 i-2}, 2 i-1, \ldots, n\right)\right. \\
& \times \frac{A_{2 i-3}^{\prime}\left(P_{2 i-1: 2}, 3, \ldots, 2 i-2\right)}{z P_{2 i-1: 2}^{2}(z)} \\
& \left.+\frac{A_{3}^{\prime}\left(P_{4: 1}, 2,3\right) \times A_{n-1}^{\prime}\left(P_{23}, 4, \ldots, n, 1\right)}{z P_{23}^{2}}\right]_{z=\infty} .
\end{aligned}
$$

\section{CONCLUSIONS}

We have proposed a new CHY integrand for the $U(N)$ nonlinear sigma model. For this new integrand, the kinematic matrix, $(\mathrm{A})_{j k}^{i j}$, is no longer antisymmetric. We have found two new factorization identities, Eqs. (37) and (38). We have written the second factorization formula in an elegant way, which only involves the generalized amplitude, $A_{q}^{\prime}$. This formula turns out to be surprisingly compact (we have checked agreement of the soft limit of this formula with Ref. [17]).

This has implications for the BCFW recursion since the two new factorization formulas can be split among even and odd subamplitudes, e.g., $A_{2 q}^{\prime} \times A_{2 m}^{\prime}$ and $A_{2 q+1}^{\prime} \times A_{2 m+1}^{\prime}$ respectively. Using this we are able to give a physical meaning to the odd subamplitudes as boundary contributions under such recursions.

Work in progress [14] is going to present a new recurrence relation and investigate its connection to Berends-Giele [18-22] currents and Bern-CarrascoJohansson (BCJ) numerators [23-25]. Similar relations for other effective field theories $[13,17,22]$ are expected and will be another focus.

Despite similarities between the three-point amplitudes with the Feynman vertices obtained in Ref. [26], the construction presented here is different. For example, the numerators found in Eq. (32) are not reproduced by the Feynman rules found in Ref. [26]. Understanding the relationship between the formalisms would be interesting.

\section{ACKNOWLEDGMENTS}

Numerous discussions with J. Bourjaily and P. H. Damgaard are gratefully acknowledged. We thank C. Vergu for pointing out a useful identity. This work was supported in part by the Danish National Research Foundation (DNRF91) and H. G. in part by the University Santiago de Cali (USC). 
[1] F. Cachazo, S. He, and E. Y. Yuan, Scattering equations and Kawai-Lewellen-Tye orthogonality, Phys. Rev. D 90, 065001 (2014); Scattering of Massless Particles in Arbitrary Dimensions, Phys. Rev. Lett. 113, 171601 (2014); Scattering of massless particles: Scalars, gluons and gravitons, J. High Energy Phys. 07 (2014) 033.

[2] L. Mason and D. Skinner, Ambitwistor strings and the scattering equations, J. High Energy Phys. 07 (2014) 048.

[3] N. Berkovits, Infinite tension limit of the pure spinor superstring, J. High Energy Phys. 03 (2014) 017; H. Gomez and E. Y. Yuan, $N$-point tree-level scattering amplitude in the new Berkovits' string, J. High Energy Phys. 04 (2014) 046.

[4] N. E. J. Bjerrum-Bohr, P. H. Damgaard, P. Tourkine, and P. Vanhove, Scattering equations and string theory amplitudes, Phys. Rev. D 90, 106002 (2014).

[5] L. Dolan and P. Goddard, Proof of the formula of Cachazo, He and Yuan for Yang-Mills tree amplitudes in arbitrary dimension, J. High Energy Phys. 05 (2014) 010.

[6] C. Baadsgaard, N. E. J. Bjerrum-Bohr, J. L. Bourjaily, and P. H. Damgaard, Integration rules for scattering equations, J. High Energy Phys. 09 (2015) 129; Scattering equations and Feynman diagrams, J. High Energy Phys. 09 (2015) 136.

[7] N. E. J. Bjerrum-Bohr, J. L. Bourjaily, P. H. Damgaard, and B. Feng, Analytic representations of Yang-Mills amplitudes, Nucl. Phys. B913, 964 (2016); Manifesting color-kinematics duality in the scattering equation formalism, J. High Energy Phys. 09 (2016) 094.

[8] F. Cachazo and H. Gomez, Computation of contour integrals on $\mathcal{M}_{0, n}$, J. High Energy Phys. 04 (2016) 108.

[9] C. Cardona, B. Feng, H. Gomez, and R. Huang, Cross-ratio identities and higher-order poles of CHY-integrand, J. High Energy Phys. 09 (2016) 133.

[10] C. Baadsgaard, N. E. J. Bjerrum-Bohr, J. L. Bourjaily, P. H. Damgaard, and B. Feng, Integration rules for loop scattering equations, J. High Energy Phys. 11 (2015) 080; New Representations of the Perturbative S-Matrix, Phys. Rev. Lett. 116, 061601 (2016).

[11] H. Gomez, $\Lambda$ scattering equations, J. High Energy Phys. 06 (2016) 101.

[12] C. Cardona and H. Gomez, Elliptic scattering equations, J. High Energy Phys. 06 (2016) 094; C. Cardona and H. Gomez, CHY-graphs on a torus, J. High Energy Phys. 10 (2016) 116; H. Gomez, S. Mizera, and G. Zhang, CHY loop integrands from holomorphic forms, J. High Energy Phys. 03 (2017) 092.
[13] F. Cachazo, S. He, and E. Y. Yuan, Scattering equations and matrices: From Einstein to Yang-Mills, DBI and NLSM, J. High Energy Phys. 07 (2015) 149.

[14] H. Gomez and A. Helset, Scattering Equations and Factorization of Amplitudes II: Effective Field Theories, arXiv:1902.02633.

[15] N. E. J. Bjerrum-Bohr, P. H. Damgaard, and H. Gomez, New factorization relations for Yang Mills amplitudes, arXiv:1810.05023; H. Gomez, Scattering equations and a new factorization for amplitudes I: Gauge theories, arXiv: 1810.05407.

[16] R. Britto, F. Cachazo, B. Feng, and E. Witten, Direct Proof of Tree-Level Recursion Relation in Yang-Mills Theory, Phys. Rev. Lett. 94, 181602 (2005).

[17] F. Cachazo, P. Cha, and S. Mizera, Extensions of theories from soft limits, J. High Energy Phys. 06 (2016) 170.

[18] F. A. Berends and W. T. Giele, Recursive calculations for processes with $n$ gluons, Nucl. Phys. B306, 759 (1988).

[19] G. Chen and Y. J. Du, Amplitude relations in nonlinear sigma model, J. High Energy Phys. 01 (2014) 061; Y. J. Du and C. H. Fu, Explicit BCJ numerators of nonlinear simga model, J. High Energy Phys. 09 (2016) 174.

[20] I. Low and Z. Yin, Ward Identity and Scattering Amplitudes for Nonlinear Sigma Models, Phys. Rev. Lett. 120, 061601 (2018).

[21] K. Kampf, J. Novotny, and J. Trnka, Tree-level amplitudes in the nonlinear sigma model, J. High Energy Phys. 05 (2013) 032.

[22] S. Mizera and B. Skrzypek, Perturbiner methods for effective field theories and the double copy, J. High Energy Phys. 10 (2018) 018.

[23] Z. Bern, J. J. M. Carrasco, and H. Johansson, New relations for gauge-theory amplitudes, Phys. Rev. D 78, 085011 (2008).

[24] Y. J. Du and Y. Zhang, Gauge invariance induced relations and the equivalence between distinct approaches to NLSM amplitudes, J. High Energy Phys. 07 (2018) 177.

[25] J. J. M. Carrasco, C. R. Mafra, and O. Schlotterer, Abelian Z-theory: NLSM amplitudes and $\alpha$-corrections from the open string, J. High Energy Phys. 06 (2017) 093; Semi-abelian Z-theory: NLSM $+\phi^{3}$ from the open string, J. High Energy Phys. 08 (2017) 135.

[26] C. Cheung and C.H. Shen, Symmetry for FlavorKinematics Duality from an Action, Phys. Rev. Lett. 118, 121601 (2017); C. Cheung, G. N. Remmen, C. H. Shen, and C. Wen, Pions as gluons in higher dimensions, J. High Energy Phys. 04 (2018) 129. 